\title{
On the Node Density Limits and Rate-Delay-Energy Tradeoffs in Ad Hoc Nanonetworks with Minimum Energy Coding
}

\author{
Murat Kocaoglu Derya Malak \\ Next-generation and Wireless Communications Laboratory (NWCL) \\ Department of Electrical and Electronics Engineering \\ Koc University, Istanbul, Turkey \\ Email:\{mkocaoglu,dmalak\}@ku.edu.tr
}

\begin{abstract}
Ad-hoc nanonetworks are collections of nanonodes without central controller units, and are the most promising network architectures in nano communications. Derivation of maximum nanonode density can pave the way for determining the capacity of ad-hoc nanonetworks. We consider ad-hoc nanonetworks with minimum energy coding (MEC). Maximum nanonode density for reliable communication in an ad-hoc nanonetwork without any medium access control is derived, and density dependent reliability analysis is conducted. Rate-delay-energy tradeoffs are also investigated with achievable rates, with constant codebook size and constant Hamming distance, separately.
\end{abstract}

\section{INTRODUCTION}

Nanonetworks, composed of a large number of nanonodes are believed to find application in various fields, from healthcare to industrial applications [1]. In nano-EM communications, electromagnetic waves are used as information carrier. Hence, it is similar to classical communications used in our daily lives. Due to extremely small size, new materials specific to nano-domain, such as carbon nanotubes (CNTs), should be used. In order to address novel characteristics of nanodevices, development of new communication techniques is required.

In the literature, there exist several studies on nanonetworks [2]-[5]. The first work on ad-hoc nanonetworks is presented in [4]. Authors investigated the challenges to be addressed for the realization of ad-hoc nanonetworks. In [5], authors showed that without any medium access control mechanism, low weight channel codes can be used for communication in nanonetworks, together with OOK modulation, without considering any specific coding scheme. To address the severe energy efficiency requirement, we proposed novel minimum energy channel codes (MEC), for reliable nanoscale communications in [3]. We have considered a nanosensor network, in which time or frequency resources are orthogonally allocated, and investigated its feasibility. However, nanonetworks with

This work was supported in part by the Turkish Scientific and Technical Research Council under grant \#109E257, by the Turkish National Academy of Sciences Distinguished Young Scientist Award Program (TUBA-GEBIP), by IBM through IBM Faculty Award, and by Turk Telekom under Grant Number $11315-04$ central controller units might not always be feasible. Therefore, investigation of ad-hoc nanonetworks is an open research problem requiring further investigation. To the best of authors' knowledge, no channel coding scheme, guaranteeing reliability is proposed for ad-hoc nanonetworks.

In this paper, we propose using MEC for reliable communication in ad-hoc nanonetworks. A probabilistic analysis is conducted to show that, reliable communication is possible in ad-hoc nanonetworks without any medium access scheme. We investigate the maximum node density, that allows perfectly reliable communication, with increasing code distance. Relation between nanonode density of ad-hoc network and reliability is revealed through simulations. We also investigate ratedelay-energy tradeoffs of ad-hoc nanonetworks with MEC. It is shown that, for maximally dense networks, increasing delay using larger source set cardinality increases the rate. Achievable rates for sufficiently large code distance and source set cardinality are also analytically derived.

The rest of this paper is organized as follows. In Sec. II, a review of minimum energy coding (MEC) is provided and important results are underlined. In Sec. III, the ad-hoc nanonetwork description is provided with the assumptions on the nanonodes and the network. Later, we analyze the upper limit of node density for the nanonetwork together with its effects on reliability in Sec. IV. Besides, rate-delay-energy tradeoffs for constant code distance and constant source set cardinality are investigated. Conclusions are given in Sec. V

\section{Minimum Energy Codes}

In our previous work [3], we have proposed novel minimum energy codes with controllable reliability to be used for nano communications. In this section, we review the basic results obtained in our previous work and highlight the parts that will be used in this paper.

In coding theory, a codebook is any selection of fixed length codewords, $c_{i}$ ' $s$, mapped to source symbols. Hamming weight of a codeword $c_{i}$ is denoted with $w_{i}$, and is defined as the number of non-zero entries in the codeword. Hamming weight is equivalent to the number of 1 's in the codeword for binary 
codes. Hamming distance between two codewords is defined as the number of bits that they differ in, whereas Hamming distance of a code, is the minimum of all Hamming distances.

In this paper, $p_{\max }, d$ and $M$ represent the probability of most likely source outcome, code distance, and source set cardinality, respectively. As a precondition of the proposed minimum energy codes, an OOK modulation scheme with $l$ carriers, each located at allowable frequency bands in the $\mathrm{THz}$ channel is proposed. In this paper, we assume $l=1$ for simplicity. The analysis can be easily generalized to any $l$.

\section{A. Minimum Expected Weight}

In classical communication scenarios, both source coding and channel coding schemes are employed in general. Source coding reduces redundancies in the source symbols, whereas channel codes are used to add redundancy to combat channel noise and other detrimental factors. To keep complexity low, our proposed technique do not employ any source coding mechanism. Another advantage of not using source coding is that, codewords to be used are not equiprobable in general, which can be exploited to reduce energy consumption on the average, using minimum energy codes. Our proposed minimum energy codes, MEC, yields the minimum average code weight, depending on the source distribution and desired reliability via Hamming distance. From [3], we know that

$$
\min (E(w))= \begin{cases}\left(1-p_{\max }\right) d, & p_{\max }>\frac{1}{2}, \\ \frac{d}{2}, & p_{\max }<\frac{1}{2}, \text { if } d \text { even } \\ \left\lceil\frac{d}{2}\right\rceil-p_{\max }, & p_{\max }<\frac{1}{2}, \text { if } d \text { odd }\end{cases}
$$

\section{B. Codebook generation}

Although different approaches exist to generate a minimum energy codebook, a simple and tractable scheme is proposed. With this approach, minimum codeword length is obtained as

$$
n_{\text {min }}=d+(M-2)\left\lceil\frac{d}{2}\right\rceil .
$$

To ease the analysis, we stick with the assumptions in our previous work, and assume $p_{\max }>0.5$ and $d$ is even.

A sample codebook generation can be given as follows: If $p_{\max }$ is greater than 0.5 , all 0 codeword should exist in the codebook and be mapped to the most probable source outcome. In order to assure minimum Hamming distance, other codewords should be weight $-d$, since code distance is desired to be $d$. Codeword selection is more straightforward if $p_{\max }<0.5$. Let $\mathbf{1}_{k}$ and $\mathbf{0}_{k}$ represent a length $-k$ block of ones, and a length $-k$ block of zeros, respectively. Then the rows of the following matrix are codewords generated by the proposed mechanism for $p_{\max }<0.5$ and right hand side of the equation yields the case for $d=4$ :

$$
\left\{\begin{array}{ccc}
\mathbf{0}_{0} & \mathbf{1}_{\frac{d}{2}} & \mathbf{0}_{n-\frac{d}{2}} \\
\mathbf{0}_{\frac{d}{2}} & \mathbf{1}_{\frac{d}{2}} & \mathbf{0}_{n-d} \\
\mathbf{0}_{d} & \mathbf{1}_{\frac{d}{2}} & \mathbf{0}_{n-\frac{3 d}{2}} \\
\vdots & \vdots & \vdots
\end{array}\right\}=\left\{\begin{array}{cccccccc}
1 & 1 & 0 & 0 & \cdots & 0 & 0 & 0 \\
0 & 0 & 1 & 1 & \cdots & 0 & 0 & 0 \\
\vdots & \vdots & & & & & & \vdots \\
0 & 0 & 0 & 0 & \cdots & 0 & 1 & 1
\end{array}\right\}
$$

Note that $\mathbf{0}_{0}$ represents null vector with dimension 0 .

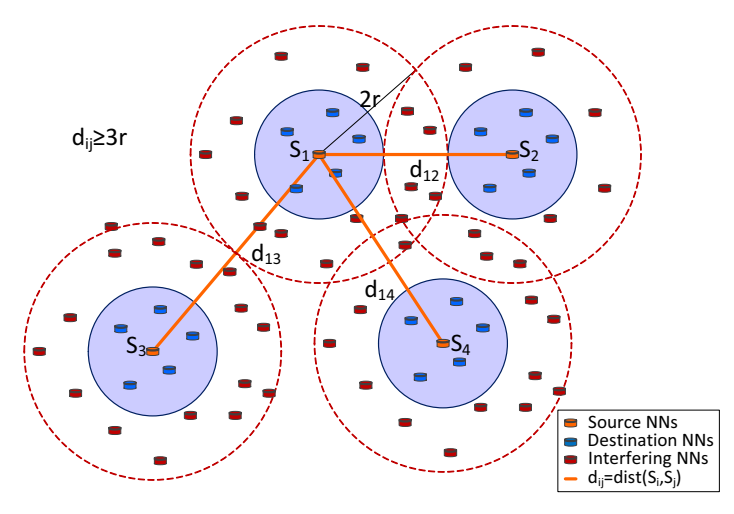

Fig. 1. Ad-hoc Nanonetwork with potential destinations and potential interfering nanonodes

\section{NANOSCALE AD-HOC NETWORKS}

The term ad-hoc network is in general used to represent networks with no central controllers, opposed to cellular networks. These networks are studied in the literature for many years, under different assumptions such as mobile nodes, or multi hop communications. Ad-hoc nanonetworks are first considered in [4]. In that work, authors illustrate the challenges for the realization of CNT-based nanoscale ad-hoc networks.

The ad-hoc network considered in this work is given in Fig. 1. Nanonodes do not forward codewords, different from classical ad-hoc networks, due to complexity considerations. A source nanonode attempts communication with a nanonode within its range of operation, $r$. Transmission range is used to obtain the maximum node density in the ad-hoc nanonetwork. Destination nodes attempt to decode as many codewords as possible, without considering the source nanonode. Hence, no link is established between source and destination nanonodes. Such a scenario applies to nanosensor networks, in which a destination nanonode collects as much data as possible.

Errors are assumed to be due to collisions only, which is justified by keeping symbol error probability below $10^{-9}$, by choosing the proper transmission distance for fixed transmission power. Similar to our previous work [3], a CNT antenna is set to dissipate a power of $5 \mu \mathrm{W}$, which is currently the largest power level a CNT antenna can support [6]. In the symbol error probability calculation, only path loss and thermal noise are included as detrimental factors. Molecular absorption and molecular noise is ignored, since the selected modulation scheme uses allowable frequency windows in the $\mathrm{THz}$ band, in which molecular effects are negligible. Nanonodes in the interference range are assumed to be distributed within an area of $2 \pi(2 r)^{2}$, where $r$ is the maximum distance, at which error probability of OOK modulated symbols does not exceed $10^{-9}$. Since the transmission range of a nanonode is $r$, a destination within range can be compromised by nodes within $2 r$ range. Hence, maximum number of nanonodes supplied by MEC should be distributed within a range of $2 r$. Choosing the band of operation as $1 \mathrm{THz}$ and bandwidth as $10 \mathrm{GHz}, r$ is easily found to be equal to $10^{-3}$ meters. 


\section{Limits AND TRADEOFFS IN AD-HOC NANONETWORKS WITH MEC}

Nanonetworking is a promising field of research. Feasibility of nanonetworks depends on energy efficiency, robustness against highly dynamic conditions and self sustainability. It might not always be possible to implement central controller units in certain nanonetworking scenarios. In such cases, the communication scheme should provide reliable access to the channel. However, using complex medium access techniques is not feasible in nanonodes due to the limited complexity. Moreover, popular spread spectrum multiple access techniques such as CDMA cannot be used, since the THz channel shows frequency selective characteristics, which would result severe distortion of the signal, when passed through the channel. It is proposed in [5] that, using low weight channel codes might drop the necessity of a medium access scheme. In this paper, the same idea is employed for the analysis of ad-hoc nanonetworks using MEC. It is expected that, as more and more nodes communicate with each other within the transmission range of a source node, successful communication probability decreases. For nanonetworks using MEC, the following question is answered: "What is the maximum node density an adhoc nanonetwork with MEC can supply without compromising reliability?". Discovering the maximum nanonode density is important, since it indicates the communication and computation capability of a nanonetwork within a given amount of area. It is shown that, reliable communication can be achieved in ad-hoc nanonetworks satisfying $s<1 / p$, where $p$ is the transmission probability and $s$ is the number of nanonodes within the interference range of source. Furthermore, ratedelay tradeoffs for ad-hoc nanonetworks are investigated. The question, "What is the achievable rate in ad-hoc nanonetworks for sufficiently large delay?", is answered. It is shown that, different rate expressions emerge for constant $M$ or $d$.

\section{A. Maximum Node Density vs. Reliability}

First, we analyze the successful codeword decoding probability at the destination nanonode. It is assumed that a nanonode attempts transmission with probability of $p$. Instead of having nanonodes transmitting continuously, nodes transmitting only when they require, not only reduces interference, but also the energy consumption. $s$ is the number of nodes within the interference range of the source nanonode. It is assumed that $s$ neighbor nanonodes exist within a distance of $2 r$, when nanonodes are uniformly distributed with density of

$$
\rho=\frac{s+1}{2 \pi(2 r)^{2}}
$$

To assure reliability, the correct codeword decoding probability is derived. Probability that the transmitted bit is 1 is

$$
\begin{aligned}
\eta_{1} & =\sum_{i=1}^{M} p\left(1 \mid c_{i}\right) p\left(c_{i}\right) \\
& =\sum_{i=1}^{M} \frac{w_{i}}{n_{\min }} p_{i}=\frac{E(w)}{n_{\min }}
\end{aligned}
$$

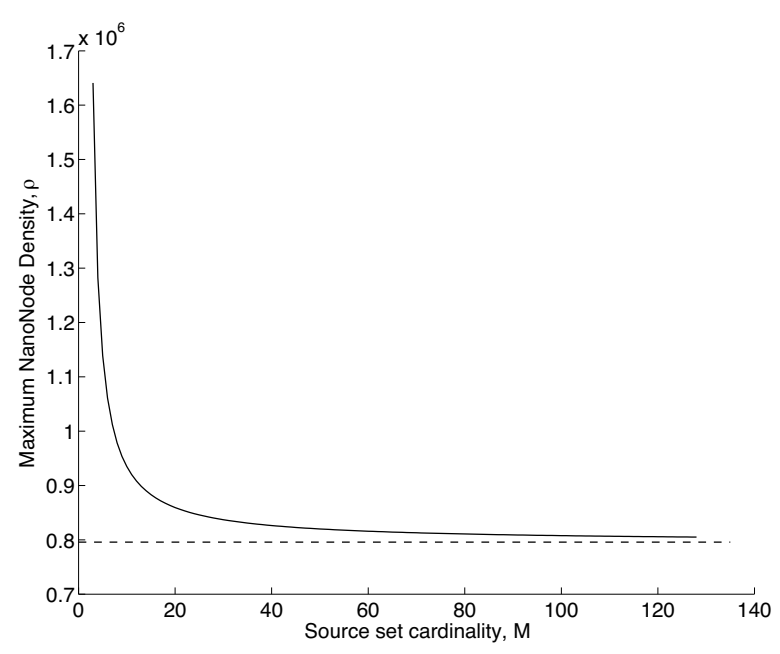

Fig. 2. Maximum allowed node density vs. source set cardinality for $\mathrm{p}=0.05$. Limiting density is also indicated at $\rho_{\text {lim }}=1 /\left(p A_{t r}\right)$

using the law of total probability, where $w_{i}$ is the weight of the codeword $c_{i}$ that is mapped to source with probability $p_{i}$. In order the transmitted bit to be received by the destination properly, interfering nodes should either transmit 0 bit or not transmit at all. The probability that there is at least single node transmitting bit 1 within the interference range, i.e., $p_{x}$ is

$$
\begin{aligned}
p_{x} & =1-\sum_{i=0}^{s}\left(\begin{array}{l}
s \\
i
\end{array}\right)\left(p \eta_{0}\right)^{i}(1-p)^{(s-i)} \\
& =1-\left(1-p\left(1-\eta_{0}\right)\right)^{s}
\end{aligned}
$$

where $\eta_{0}=1-\eta_{1}$, i.e., the transmission probability of bit 0 . Collision probability is non-zero only if 0 is transmitted by the source. If 1 is transmitted, as interference caused by other nodes cannot flip this bit, collision cannot occur. As a result, collision probability of the nanonode can be calculated as

$$
p_{c}=\eta_{1} 0+\eta_{0} p_{x}=\eta_{0}-\eta_{0}\left(1-p\left(1-\eta_{0}\right)\right)^{s} .
$$

As previously discussed, symbol error probability is assured to be less than $10^{-9}$, by choosing the transmission range. Hence, collision can be considered as the only error source, leading to simplified analysis of correct decoding probability. Eventually, since a maximum of $\left\lfloor\frac{d-1}{2}\right\rfloor$ collisions can be corrected, correct decoding probability at the destination, $\xi_{d}$, can be written as

$$
\xi_{d}=\sum_{i=0}^{\left\lfloor\frac{d-1}{2}\right\rfloor}\left(\begin{array}{c}
n_{\min } \\
i
\end{array}\right) p_{c}^{i}\left(1-p_{c}\right)^{n_{m i n}-i}
$$

A similar expression as (9) is obtained in our previous work [3], and using a similar analysis, it can be concluded that, $\xi_{d}$ converges to 1 with increasing Hamming distance, if $p_{c}<$ $1 / M$. In other words, reliable communication can be achieved if collision probability is less than the inverse of source set cardinality. For simplicity, assume $p_{\max }<0.5$ and $d$ even. 


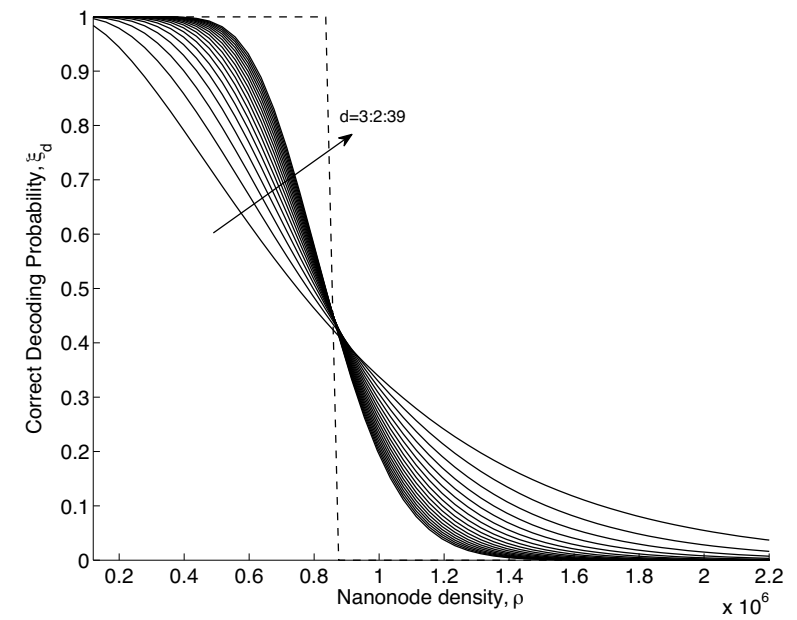

Fig. 3. Correct decoding probability vs number of source nodes for $\mathrm{p}=0.05$

Using (1), (2), (5) and (8), we reach to the condition

$$
\begin{array}{r}
1-\left[1-\frac{p}{M}\right]^{s}<\frac{1}{M-1} \\
p<M\left[1-\left(\frac{M-2}{M-1}\right)^{1 / s}\right] .
\end{array}
$$

(11) gives the relation between $p, M$, and $s$, i.e. node transmission probability, source set cardinality, and number of neighboring nodes, for which reliable communication is possible with MEC. It is easily seen that, expression on the right hand side of (11) decreases with increasing M. Hence, we can take the limit of (11) to obtain an upper bound for $p$, valid for any $M$, to achieve reliable communication.

$$
\begin{aligned}
p_{\text {lim }} & <\lim _{M \rightarrow \infty} M\left(1-\left(1-\frac{1}{M-1}\right)^{\frac{1}{s}}\right) \\
& =\lim _{M \rightarrow \infty} M\left(1-\sum_{k=0}^{\infty} C\left(\frac{1}{s}, k\right)\left(\frac{-1}{M-1}\right)^{k}\right) \\
& =\lim _{M \rightarrow \infty} \sum_{k=1}^{\infty} C\left(\frac{1}{s}, k\right) \frac{(-1)^{k+1} M}{M-1^{k}}=\frac{1}{s},
\end{aligned}
$$

where the general binomial coefficient for $k>0$ is

$$
C\left(\frac{1}{s}, k\right)=\frac{1}{k !}\left(\frac{1}{s}\right)\left(\frac{1}{s}-1\right) \cdots\left(\frac{1}{s}-k+1\right) \text {. }
$$

It is concluded that $p<1 / s$ is the required condition, satisfying (11) for any $M$. Hence, MEC supplies an ad-hoc nanonetwork having $s$ neighbor nodes, only if nodes transmit with probability less than $1 / s$. Interpreting the result from the other side leads to the desired bound for maximum node density. Maximum number of nodes within the interference range should be less than $1 / p$. This corresponds to the density

$$
\rho_{\max }=\frac{\lfloor 1 / p\rfloor+1}{A_{t r}}=\frac{\lfloor 1 / p\rfloor+1}{8 \pi} \times 10^{6} \mathrm{~m}^{-2},
$$

where $A_{t r}$ is the transmission area. The result is verified by simulation results as illustrated in Fig. 2.

Variation of reliability with respect to node density is shown in Fig. 3. As claimed in the analytical evaluations, reliability can be increased by increasing Hamming distance of the channel code if node density is below a threshold, corresponding to $1 / p$ number of neighboring nodes. This shows that, MEC with large delays can compensate the absence of a medium access control scheme up to nanonode density of $\rho_{\max }$.

\section{B. Rate Delay Energy Tradeoffs in Ad-Hoc Nanonetworks}

Rate-delay tradeoff of networks is vastly investigated in the classical communication scenarios. In most communication scenarios, capacity is achieved with infinite delay. This makes the rate-delay tradeoffs worthy of investigation, to uncover the rate achievable with finite delay. The same idea applies in nanonetworks using MEC. Different from classical scenarios, energy dissipation should also exist in this picture, as it is one of the most important metrics for nano communications. As no retransmission or channel contention exists, delay is solely due to codeword length, ignoring propagation delay, since transmission range of nanonodes is very small. Hence, delay is given as

$$
\delta=n_{\min } T,
$$

where $T$ is the symbol duration. Due to the used modulation technique, $T=10 \mathrm{ps}$. Additionally, we provide a different rate definition as

$$
R=\xi_{d} \log M
$$

(16) is the expected amount of information correctly decoded at the destination nanonode. Without an information theoretic approach, this definition provides a simple and insightful way to investigate rate-delay tradeoffs in ad-hoc nanonetworks. Average energy per codeword depends on average weight, since OOK-based modulation at available $\mathrm{THz}$ windows is used. It is formulated as,

$$
\varepsilon=E(w) P T,
$$

where $P$ is the symbol power. Due to the power limits of CNTs, $P=5 \mu W$.

As given in (2), codeword length depends on $d$ and $M$. We use two different approaches to reveal rate-delay tradeoffs. In both approaches, $p=1 / s$ is assumed.

1) Rate-Delay-Energy Tradeoffs, Constant $M$ : Increasing code distance, $d$, increases the delay via codeword length according to (2). Since increasing $d$ makes communication more reliable, rate is expected to increase with delay. The variation of rate delay and energy with increasing Hamming distance is observed in Fig. 4. Convergence of rate-delay curve to $\log M$ is expected, since

$$
\lim _{d \rightarrow \infty} R=\lim _{d \rightarrow \infty} \xi_{d} \log M=\log M .
$$

As expected code weight increases with $d$, average energy per codeword also increases. This result indicates that, larger delays via increasing Hamming distance, can make communication reliable for a maximum rate of $\log (M)$. 


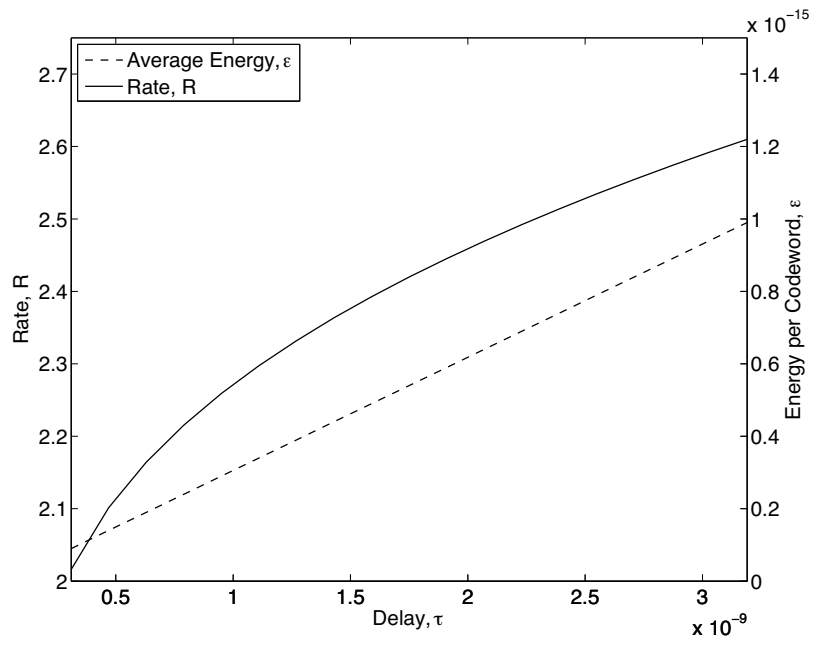

Fig. 4. Rate-delay-energy tradeoffs for $M=16, p=0.01$.

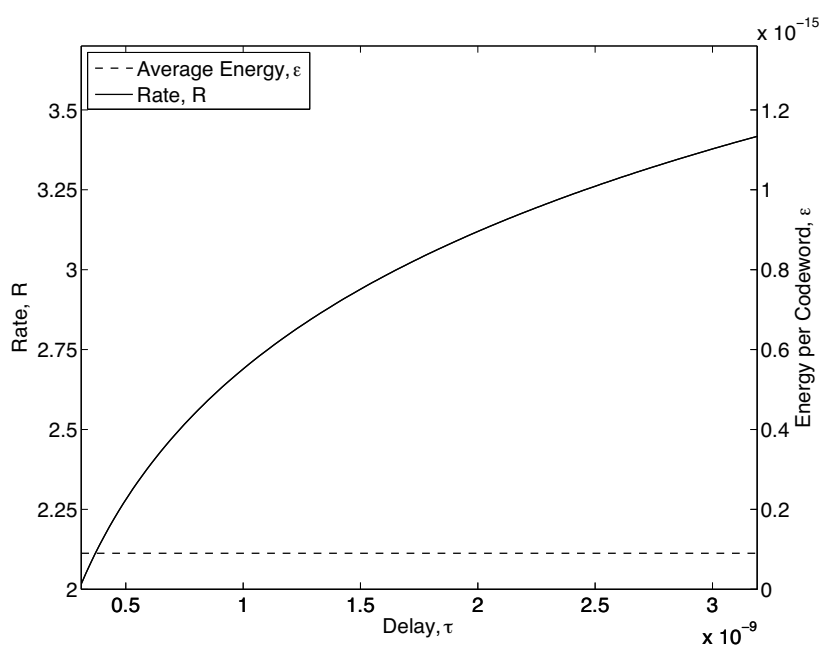

Fig. 5. Rate-delay-energy tradeoffs for $d=3, p=0.01$.

2) Rate-Delay-Energy Tradeoffs, Constant $d$ : Increasing source cardinality, $M$, increases the delay via codeword length according to (2). The question that whether increasing $M$ provides more reliable communication or not is more ambiguous. Under some simplifying assumptions, we can reach an approximate expression for collision probability, which can later be used to approximate correct decoding probability. Under the condition that $d$ is even and $p_{\max }<0.5$, using the fact that $\eta_{1}=1 / M$, for sufficiently large $s$,

$$
p_{x}=1-\left(1-\frac{p}{M}\right)^{s} \approx 1-e^{-1 / M},
$$

This leads to,

$$
p_{c} \approx \frac{M-1}{M}\left(1-e^{-1 / M}\right) .
$$

Since, $n \gg\left\lfloor\frac{d-1}{2}\right\rfloor$, from (9), we can write

$$
\begin{aligned}
\xi_{d} & \approx\left(1-p_{c}\right)^{n} \sum_{i=0}^{\left\lfloor\frac{d-1}{2}\right\rfloor}\left(\frac{n p_{c}}{1-p_{c}}\right)^{i} \frac{1}{i !} \\
& \approx e^{-\frac{d}{2}} \sum_{i=0}^{\left\lfloor\frac{d-1}{2}\right\rfloor}\left[\frac{d}{2}(M-1)\left(e^{\frac{1}{M}}-1\right)\right]^{i} \frac{1}{i !} .
\end{aligned}
$$

(22) is obtained using the expression in (20). In the limit,

$$
\lim _{M \rightarrow \infty} \xi_{d} \approx e^{-\frac{d}{2}} \sum_{i=0}^{\left\lfloor\frac{d-1}{2}\right\rfloor}\left(\frac{d}{2}\right)^{i} \frac{1}{i !}=\xi^{\prime} .
$$

From (23), as $M$ increases, $\xi_{d}$ converges to a nonzero probability value. Therefore, for very large $M$,

$$
R \approx \xi^{\prime} \log M
$$

This result reveals that, by increasing the source set cardinality at the source nanonode, one can increase communication rate and still satisfy reliable communication with high probability, for large delays. The variation of rate delay and energy with increasing source set cardinality is observed in Fig. 5, agreeing with theoretical results. Average energy does not increase with delay, since $E(w)$ does not depend on $M$.

\section{CONCLUSIONS}

In this paper, limits and tradeoffs of ad-hoc nanonetworks employing MEC are investigated. It is demonstrated that reliable communication, i.e., perfect codeword decoding can be achieved using MEC, in ad-hoc nanonetworks with a bounded number of interfering nanonodes, without any medium access control. The exact expression for decoding probability is derived, and it is concluded that nanonode density, $\rho$, should be less than the inverse of node transmission probability, $1 / p$, to achieve reliable communication. Furthermore, ratedelay-energy tradeoffs are analyzed for constant codebook size, $M$, with varying Hamming distance, $d$, and vice versa. The analyses reveal that for constant $M$, average energy and rate increase with delay. However, for constant $d$, energy dissipation remains constant, as delay increases. Additionally, it is shown that increasing source set cardinality leads to rates a constant multiple of $\log M$ for ad-hoc nanonetworks.

\section{REFERENCES}

[1] I. F. Akyildiz and J. M. Jornet, "Electromagnetic Wireless Nanosensor Networks," Nano Comm. Net. (Elsevier), vol. 1, no. 1, pp. 3-19, 2010.

[2] C. E. Koksal and E. Ekici, "A Nanoradio Architecture for Interacting Nanonetworking Tasks," Elsevier Nano Communication Networks Journal, vol. 1, pp. 6375, March 2010.

[3] M. Kocaoglu and O. B. Akan, "Minimum Energy Coding for Wireless NanoSensor Networks," to appear in IEEE INFOCOM Mini Conference 2012, Orlando, FL, USA, March 2012.

[4] B. Atakan, O. B. Akan, "Carbon nanotube-based nanoscale ad hoc networks," IEEE Comm. Magazine, vol. 48, 2010, pp. 129-135.

[5] J. M. Jornet and I. F. Akyildiz, "Low-weight channel coding for interference mitigation in electromagnetic nanonetworks in the terahertz band," Proc of IEEE International Conference on Comm., ICC, June 2011.

[6] Y. Wang et al., "Terahertz radiation from armchair carbon nanotube dipole antenna," Chinese Phys. B, vol. 18, 2009. 